Supporting Information

\title{
Decreasing Ion Diffusion Barrier Enables Superior Na-Ion Storage by Synergizing Hierarchical Architecture and Lattice Distortion
}

Min Guo, Wanyu Zhao, Huanglin Dou, Guohua Gao*, Xiaoli Zhao*, Xiaowei Yang*

School of Materials Science and Engineering, Interdisciplinary Materials Research

Center, School of Physics and Science Engineering, Tongji University, Shanghai

201804, China

\section{Corresponding Author}

*Email: yangxw@tongji.edu.cn; zx136@tongji.edu.cn; gao@tongji.edu.cn. 


\section{Experimental Sections}

Material synthesis: Zn-doped $\mathrm{Na}_{3} \mathrm{~V}_{2-\mathrm{x}} \mathrm{Zn}_{\mathrm{x}}\left(\mathrm{PO}_{4}\right)_{3}(\mathrm{x}=0,0.02,0.04,0.08,0.1)$ were prepared according to a solution freeze-drying method combined with high-temperature calcination. Firstly, stoichiometric amount of $\mathrm{H}_{2} \mathrm{C}_{2} \mathrm{O}_{4} \cdot 2 \mathrm{H}_{2} \mathrm{O}$ and $\mathrm{V}_{2} \mathrm{O}_{5}$ were dispersed in $15 \mathrm{~mL}$ deionized water and heat to $70{ }^{\circ} \mathrm{C}$ with vigorously stirred for 2 hours, then pump into $5 \mathrm{~mL}$ mixture solution of $\mathrm{Na}_{2} \mathrm{H}_{2} \mathrm{PO}_{4} \cdot 2 \mathrm{H}_{2} \mathrm{O}$ and sucrose $(0.13 \mathrm{~g})$ for other $15 \mathrm{~min}$. Subsequently, the well-responsive hydrophilic homogeneous colloid was frozen by liquid nitrogen and freezing-drying 48 hours to obtain the precursor. Finally, the precursor was preheated at $400{ }^{\circ} \mathrm{C}$ for $4 \mathrm{~h}$ followed by calcinated at $700{ }^{\circ} \mathrm{C}$ for $8 \mathrm{~h}$ in $5 \% \mathrm{H}_{2} / \mathrm{Ar}$ mixed gas to acquire the $\mathrm{Zn}$-doped NVP samples. Here, the synthesized composites $\mathrm{Na}_{3} \mathrm{~V}_{2-\mathrm{x}} \mathrm{Zn}_{\mathrm{x}}\left(\mathrm{PO}_{4}\right)_{3}(\mathrm{x}=0,0.02,0.04,0.08$, 0.1) were named NVP, NVP-Zn0.02, NVP-Zn0.04, NVP-Zn0.08, NVP-Zn0.1, respectively.

Material characterization: Scanning electron microscopy (SEM, Nova NanoSEM 450), Transmission electron microscopy (TEM, JEM-2100F II ), X-ray diffraction (XRD), Raman spectroscopy (RM1000), Fourier transform infrared spectrophotometer (FTIR, FTIR5700), X-ray photoelectron spectroscopy (XPS, ESCALAB 250Xi), thermogravimetric analysis (TGA, NETZSCH) were employed to investigated the morphology, microstructure and composition of all samples. Nitrogen physisorption measurements were performed to characterize the BET specific surface area and pore size distribution.

Electrochemical measurements: In order to make working electrodes, $70 \mathrm{wt} . \%$ active 
materials, 20 wt.\% commercial carbon nanotube (CNT), and $10 \mathrm{wt} \% \%$ polyvinylidene (PVDF) were dispersed in N-methyle-2-pyrrolidone (NMP) to form slurry. And then coated on aluminum foil. After drying 8 hours at $110{ }^{\circ} \mathrm{C}$, the aluminum foil was cut into round slices with the areal mass loading $1.2 \sim 1.5 \mathrm{mg} \mathrm{cm}^{-2} .2025$-type coin cells were assembled using the above electrodes as working electrodes and the electrolyte is $\mathrm{NaClO}_{4}(1 \mathrm{M})$ in propylene carbonate (PC) with $5.0 \% \mathrm{FEC}$, the amount of electrolyte is $160 \mathrm{uL}$. The counter electrode is modified sodium foil. ${ }^{1}$ The galvanostatic charge/discharge were conducted using a Neware battery testing system in a voltage range of $2.3 \sim 4.0 \mathrm{~V}$. Cyclic voltammetry (CV) and electrochemical impedance spectra (EIS) tests were performed on Bio-logic VMP-3 electrochemical workstation using a two-electrode system (coin cell 2025-type). The CV tests were conducted with scan rates from 0.1 to $5.0 \mathrm{mV} \mathrm{s}^{-1}$. Before testing, the coin cell was rested five hours at room temperature. The EIS measurement was tested in a fully discharged state and the frequency range was set from $100 \mathrm{kHz}$ to $10 \mathrm{mHz}$ with an amplitude of $5 \mathrm{mV}$.

\section{Calculations}

Density functional theory (DFT) analysis was applied to investigate the crystal structures of $\mathrm{Na}_{3} \mathrm{~V}_{2}\left(\mathrm{PO}_{4}\right)_{3}$. All the calculations in this paper were performed by using the Vienna ab initio simulation package (VASP), ${ }^{2}$ the exchange and correlation energy functional was treated by the Perdew-Burke-Ernzerh of variant of the generalized gradient approximation $(G G A)$ and GGA $+\mathrm{U},{ }^{3-4}$ where a $\mathrm{U}_{\text {eff }}=4 \mathrm{eV}$ was 
adapted for V. Interaction between ion and electrons were described with projector augmented wave pseudo potentials (PAW) approach, ${ }^{5-6}$ and the energy cutoff for the plane wave basis set was set to be $400 \mathrm{eV}$ and the total energy was converged to $10^{-5}$ $\mathrm{eV}$. The k-point sampling was performed using $\Gamma$ - centered $6 \times 6 \times 4$ points, which gives the total energy of a $(2 \times 2) \mathrm{Na}_{3} \mathrm{~V}_{2}\left(\mathrm{PO}_{4}\right)_{3}$ complex within $1 \mathrm{meV}$.

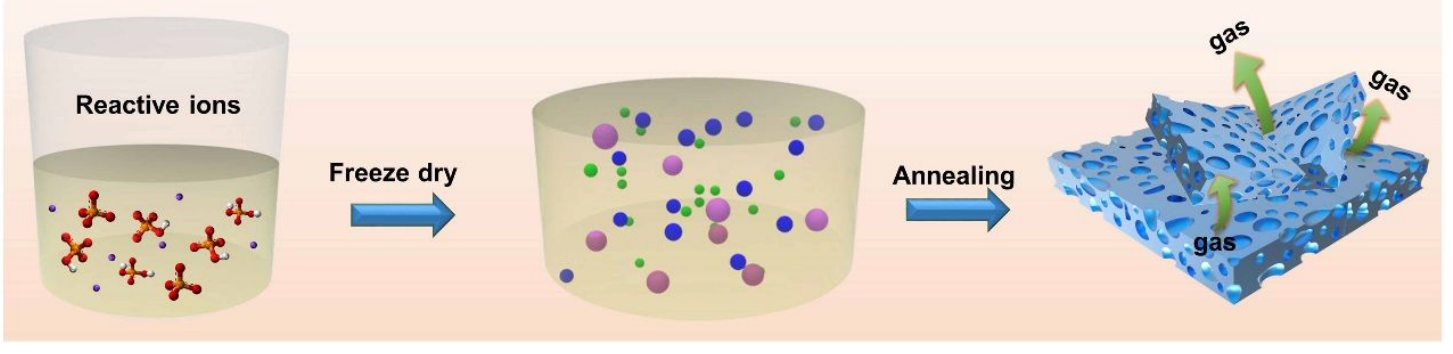

Figure S1. Schematic of the synthesis process of $\mathrm{Na}_{3} \mathrm{~V}_{2-\mathrm{x}} \mathrm{Zn}_{\mathrm{x}}\left(\mathrm{PO}_{4}\right)_{3}$ composites.
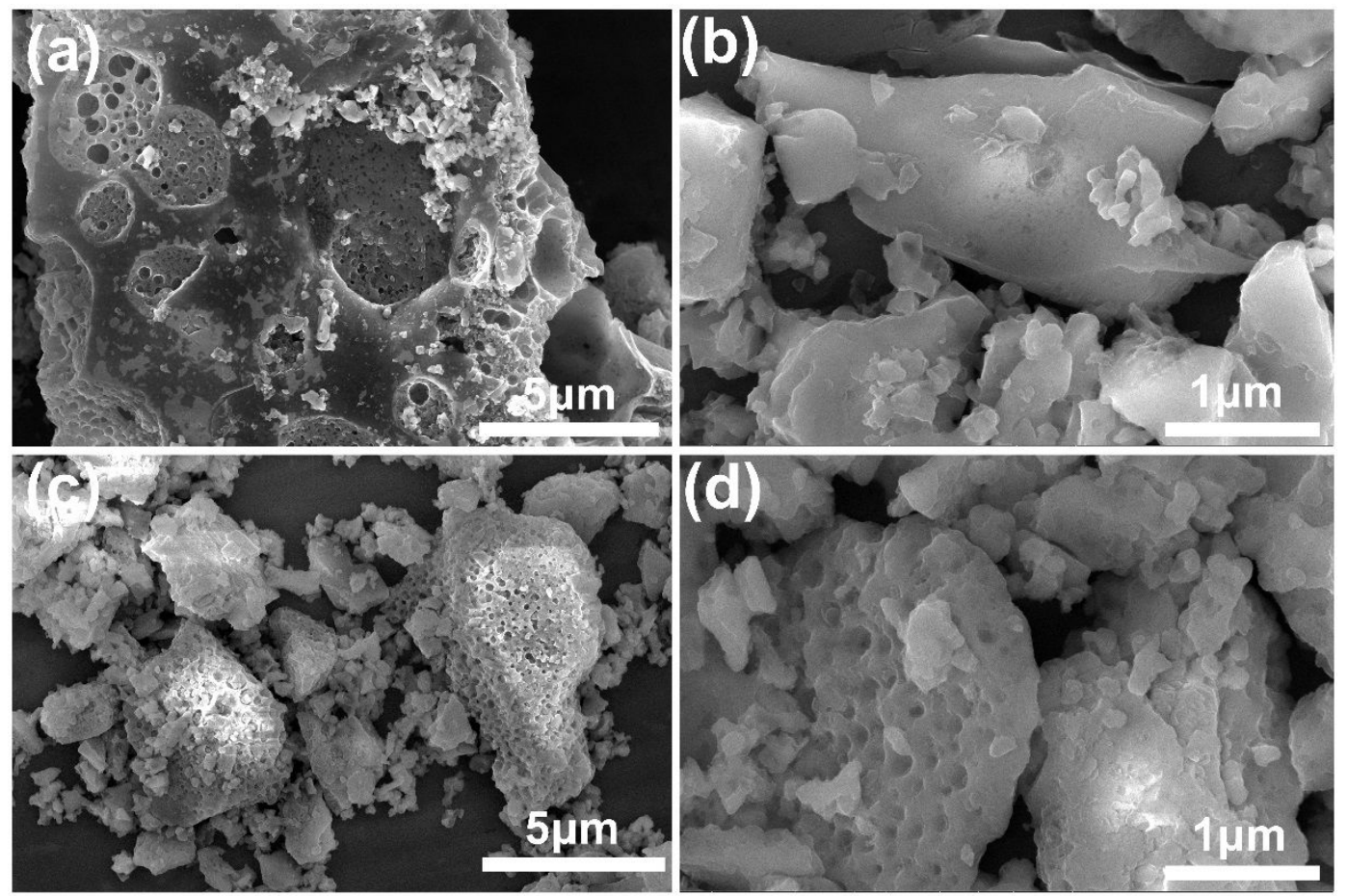
Figure S2. SEM images of (a-b) the pristine NVP and (c-d) NVP-Zn0.04 samples.

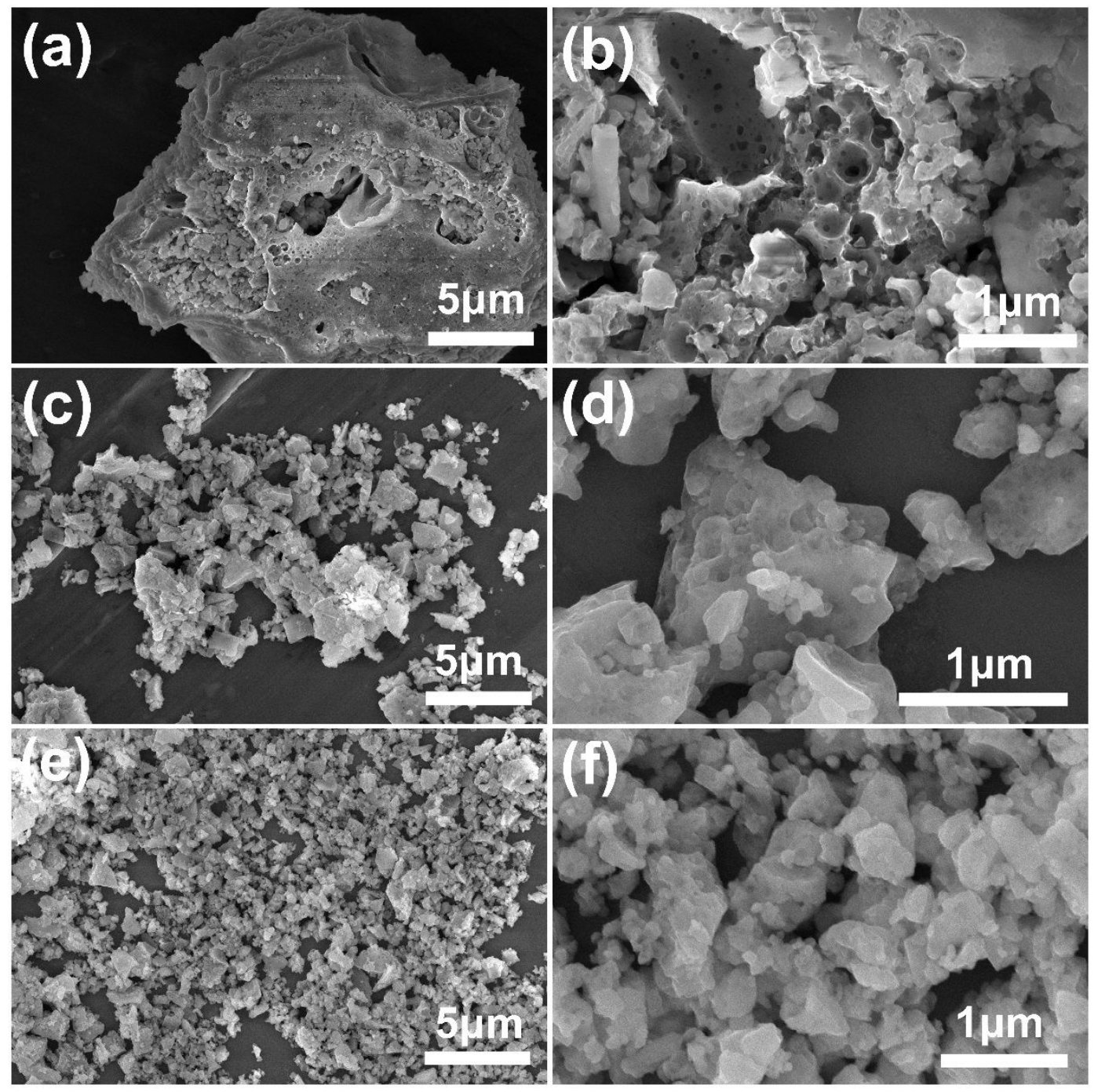

Figure S3. SEM images of (a-b) NVP-Zn0.02, (c-d) NVP-Zn0.08, (e-f) NVP-Zn0.1. 


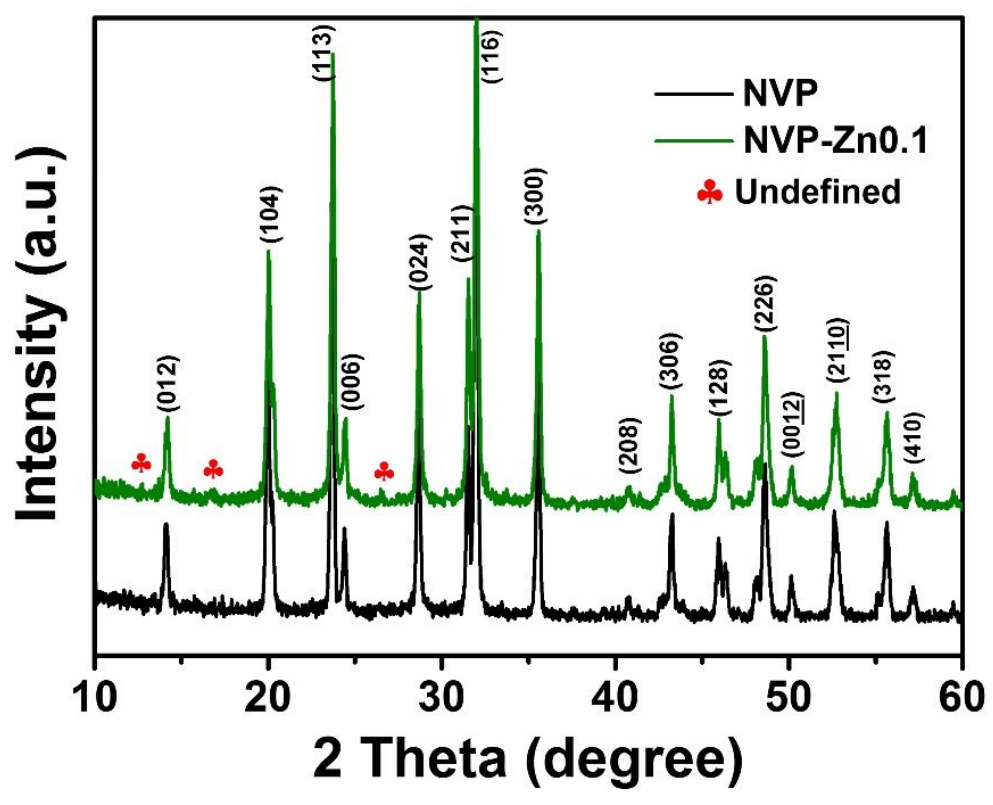

Figure S4. XRD patterns of NVP and NVP-Zn0.1 composite.
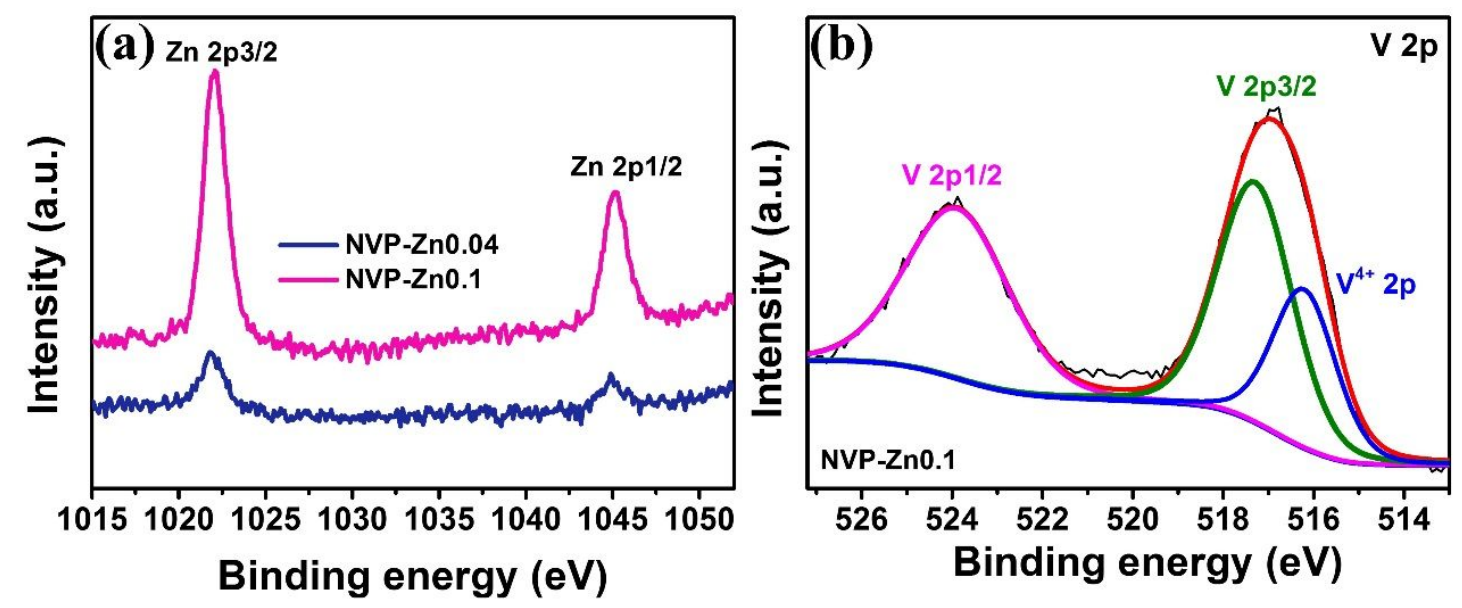

Figure S5. (a) Zn 2p spectra for NVP-Zn0.04 and NVP-Zn0.1; (b) the high-resolution of V $2 p$ spectra for NVP-Zn0.1. 


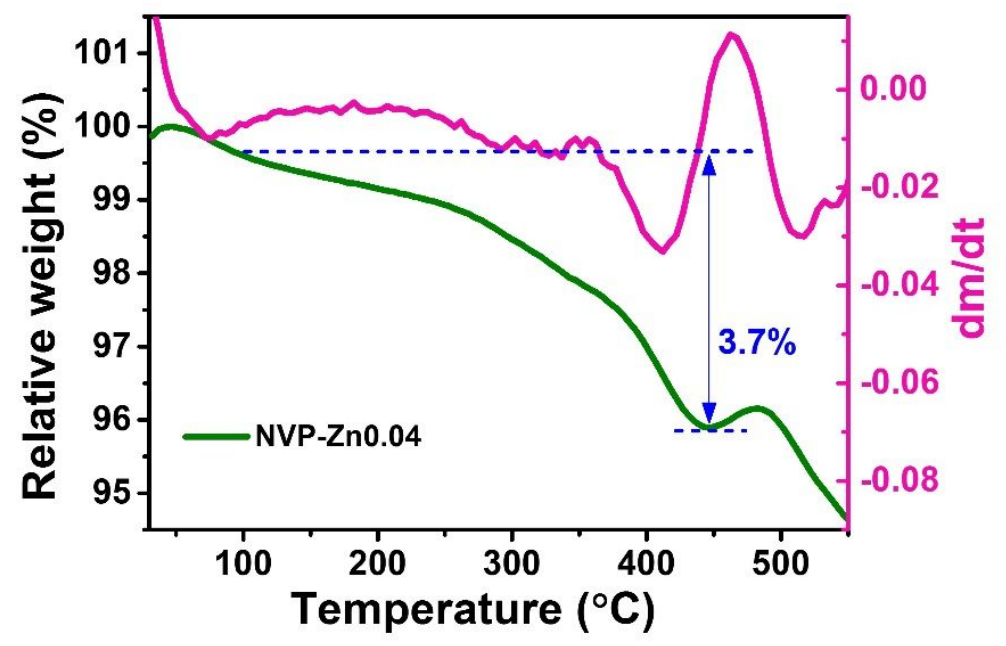

Figure S6. The TGA results of NVP-Zn0.04 sample.
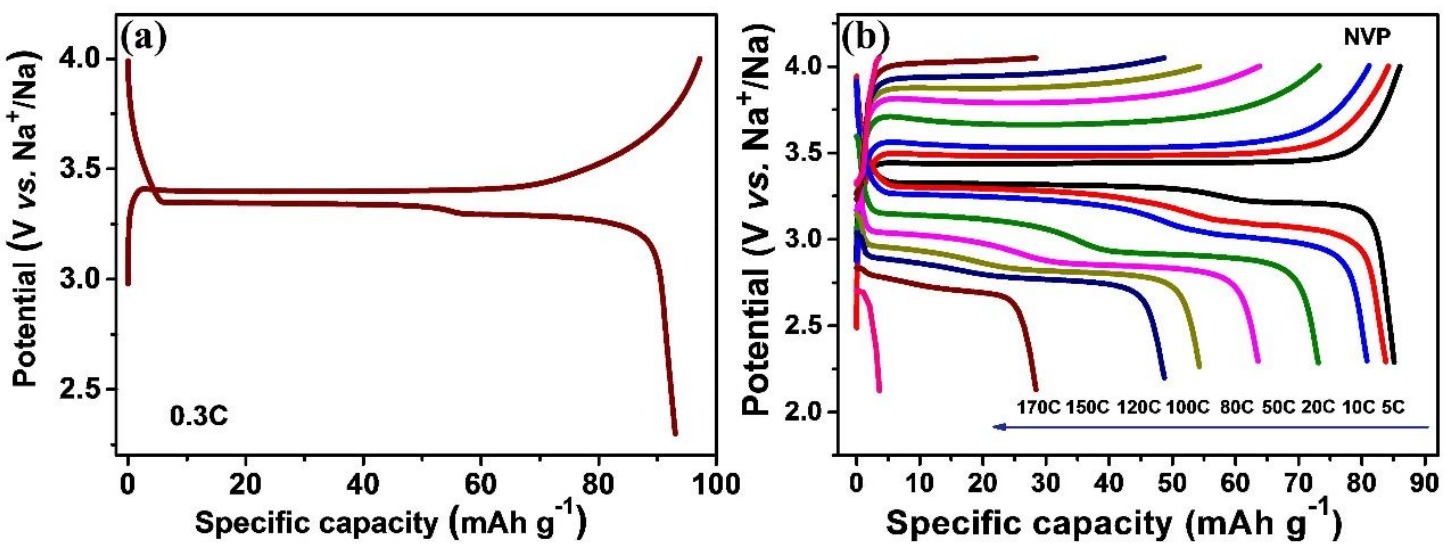

Figure S7. The charge and discharge curves of NVP (a) $0.3 \mathrm{C}$; (b) 5 to $170 \mathrm{C}$. 

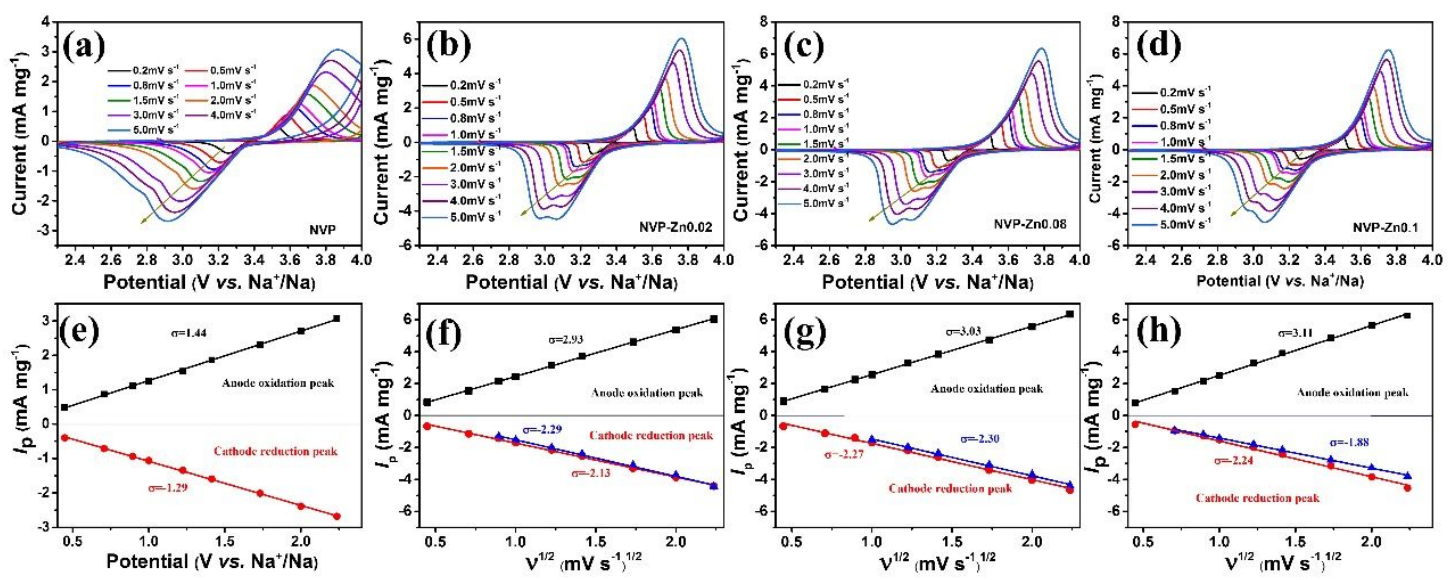

Figure S8. Cyclic voltammograms from $0.2 \sim 5.0 \mathrm{mV} \mathrm{s}^{-1}$ and the corresponding peak current against square root of scan rate: (a, e) NVP, (b, f) NVP-Zn0.02, (c, g) NVP-Zn0.08, (d, h) NVP-Zn0.1.

\section{References}

(1) Wang A. X., Hu X. F., Tang H. Q., Zhang C. Y., Liu S., Yang Y. W., Yang Q. H., Luo J. Y., Processable and moldable sodium-metal anodes, Angew. Chem., 2017, 129, 12088.

(2) Kresse G., Furthmuller J., Efficiency of ab-initio total energy calculations for metals and semiconductors using a plane-wave basis set, Computational Materials Science, 1996, 6, 15.

(3) Perdew J. P., Burke K., Ernzerhof M., Generalized Gradient Approximation Made Simple, Physical Review Letters, 1996, 77, 3865.

(4) Dudarev S. L., Botton G. A., Savrasov S. Y., Humphreys C. J., Sutton A. P., Electro-energy-loss spectra and the structural stability of nickel oxide: an LSDA+U study, Physical Review B, 1998, 57, 1505.

(5) Blochl P. E., Projector augmented-wave method, Physical Review B, 1994, 50, 
17953.

(6) Kresse G., Joubert D., From ultrasoft pseudopotentials to the projector augmented-wave method, Physical Review B, 1999, 59, 1758. 